\title{
Paclitaxel plus Ramucirumab bei Magenkarzinom
}

\author{
Patienten mit Karzinomen von Magen und ösophagogastralem Übergang \\ leben unter einer Zweitlinientherapie mit Paclitaxel plus Ramucirumab länger. \\ Ob dieser Nutzen mit Lebensqualität und Performancestatus korreliert, \\ wurde in einer Analyse der RAINBOW-Studie untersucht.
}

n der Phase-III-Studie RAINBOW hatte die Addition von Ramucirumab zu Paclitaxel in der Zweitlinientherapie von Patienten mit Karzinomen des Magens oder des ösophagogastralen Übergangs zu einem im Median 2,3 Monate länge-

ren Überleben geführt. Bei Ramucirumab handelt es sich um einen gegen den VEGFR2 („vascular endothelial growth factor receptor 2“) gerichteten humanen Antikörper. Auch die Entwicklung der Lebensqualität und des Perfor-

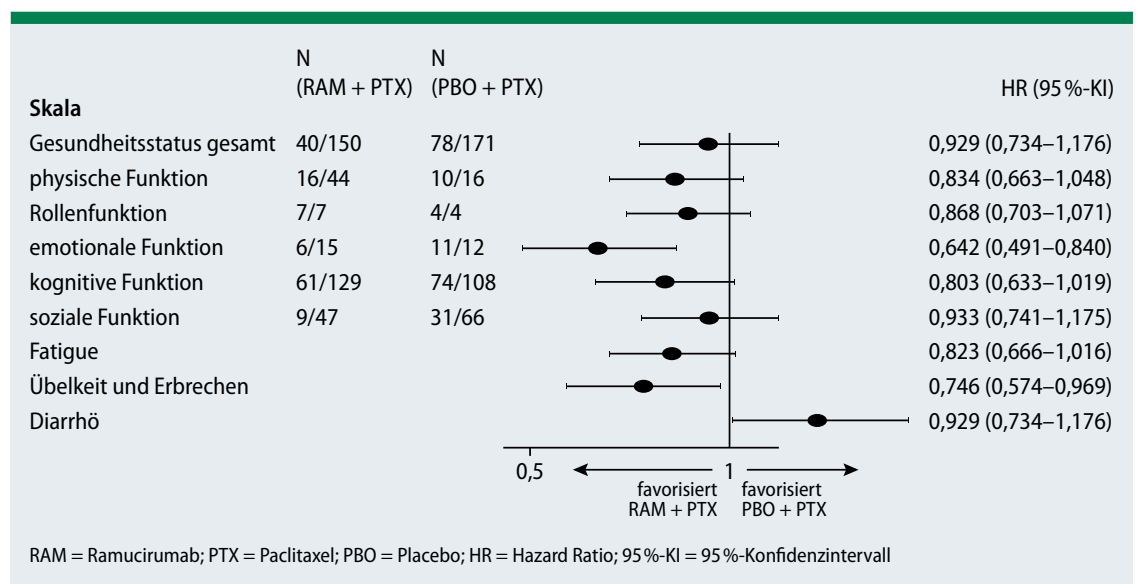

Abb. 1: RAM + PTX wurde in fast allen EORTC-QLQ-C30-Skalen favorisiert.

\section{HER2 beim Magenkarzinom mehrfach testen!}

\begin{abstract}
HER2 hat durch die Verfügbarkeit entsprechender Therapien auch beim Magenkarzinom an Bedeutung gewonnen. Ein Problem ist die Heterogenität der Expression im Primärtumor und anderen Lokalisationen.
\end{abstract}

\footnotetext{
n einer Studie wurde der HER2-Status jeweils immunhistochemisch und mittels Fluoreszenz-in-situ-Hybridisierung (FISH) bestimmt. Im 1. Teil wurden bei 183 Patienten mit nicht resektablem oder metastasiertem HER2-negativem Magenkarzinom erneut endoskopisch Biopsien entnommen, im 2. Teil wurde der HER2-Status von Metastasen oder Rezidiven bei 175 Patienten mit negativem Primärtumor untersucht.
}

Im 1. Studienteil wurden $8,7 \%(\mathrm{n}=16)$ zusätzliche Tumoren mit HER2-Überexpression identifiziert. Der positive HER2-Befund nach initial negativem Status war assoziiert mit Tumorlokalisation (diffus im Magen verteilt: 0\%, $11,7 \%$ bei anderen Lokalisationen; $\mathrm{p}=0,013$ ), Bormann-Typ ( $0 \%$ bei Typ IV, $11,7 \%$ bei allen anderen; $p=0,013$ ) und initialem immunhistochemischem HER2-Wert in der Biopsie $(6,7 \%$ bei 0 , $15,4 \%$ bei $1,25,0 \%$ bei 2 ; $p=0,028)$.

Im 2. Studienteil entdeckten die Untersucher HER2 bei 5,7\% der Patienten mit HER2-negativem Primärtumor $(\mathrm{n}=10)$, was signifikant mit der Metastasenlokalisation assoziiert war (17,2\% bei Leber-, $3,4 \%$ bei anderen Metastasen; $p=0,012$ ). 10 der 12 Patienten mit neuem Nachweis mancestatus (PS) wurden bei den Patienten untersucht. Inwieweit Ramucirumab hier ebenso Vorteile bringt, wurde mithilfe der Daten aus den Lebensqualitätsfragebögen EORTC-QLQ-C30 und EQ$5 \mathrm{D} \mathrm{zu}$ Behandlungsbeginn und zu mindestens einem weiteren Zeitpunkt in der RAINBOW-Studie untersucht.

Dabei ergab sich für Ramucirumab eine vergleichbare oder längere Zeit bis zur Verschlechterung der Lebensqualität (EORTC-QLQ-C30, Abb. 1) bzw. des PS. Die EQ-5D-Scores waren in den Gruppen vergleichbar und verschlechterten sich jeweils bei Abbruch oder Unterbrechung der Behandlung.

Fazit: Patienten mit gastroösophagealem Adenokarzinom, die in der Zweitlinie zusätzlich zu Paclitaxel Ramucirumab erhielten, lebten nicht nur länger, sondern auch ihre zu Behandlungsbeginn festgestellte Lebensqualität und der Performancestatus blieben länger stabil.

Barbara Kreutzkamp

Al-Batran SE et al. Quality-of-life and performance status results from the phase 3 RAINBOW study of ramucirumab plus paclitaxel versus placebo plus paclitaxel in patients with previously treated gastric or gastroesophageal junction adenocarcinoma. Ann Oncol. 2016; 27(4):673-9.

der HER2-Überexpression im 1. und 8 von 10 der Patienten mit HER2-positiven Metastasen/Rezidiven im 2. Studienteil erhielten Trastuzumab. Ansprechraten und progressionsfreies Überleben waren hier vergleichbar mit den Ergebnissen bei Patienten, die wegen einer von $\mathrm{Be}$ ginn an belegten HER2-Positivität diese Therapie zusätzlich zur Chemotherapie erhalten hatten.

Fazit: Es ist empfehlenswert, den HER2Status des Primärtumors und von Metastasen und Rezidiven erneut zu erheben, auch wenn die initiale Bestimmung negativ ausgefallen ist.

Friederike Klein

Park SR et al. Extra-gain of HER2-positive cases through HER2 reassessment in primary and metastatic sites in advanced gastric cancer with initially HER2-negative primary tumours: Results of GASTric cancer HER2 reassessment study 1 (GASTHER1). Eur J Cancer. 2016;53:42-50. 